\title{
Evaluación de Alternativas de Aprovechamiento Energético y Bioactivo de la Cáscara de Plátano
}

\author{
Andrés F. Rojas ${ }^{(1) \star}$, Sneyder Rodríguez-Barona ${ }^{(1)}$ y Jairo Montoya ${ }^{(2)}$ \\ (1) Facultad de Ingeniería y Arquitectura. Departamento de Ingeniería Química, Universidad Nacional de Colombia Sede \\ Manizales, Manizales, Caldas Colombia, (e-mail: anfrojasgo@unal.edu.co; snrodriguezba@unal.edu.co) \\ (2) Facultad de Ciencias Básicas y Tecnológicas. Programa de Química, Universidad del Quindío, Armenia, Quindío, \\ Colombia. (e-mail: jmontoya@uniquindio.edu.co)
}

* Autor a quien debe ser dirigida la correspondencia.

Recibido Oct. 30, 2018; Aceptado Ene. 15, 2019; Versión final Mar. 5, 2019, Publicado Oct. 2019

\section{Resumen}

El objetivo de este trabajo fue evaluar por simulación dos alternativas de aprovechamiento de la cáscara de plátano hartón verde para la generación de energía eléctrica y la obtención de compuestos polifenólicos. Se determinó la viabilidad técnica, económica y ambiental de los procesos; la simulación se alimentó con datos experimentales obtenidos en la caracterización de cáscara de plátano respecto a su análisis próximo, elemental, estructural, termogravimétrico, contenido de fenólicos totales y capacidad antioxidante. Se encontró que la cáscara puede ser empleada para la generación de energía a bajo costo y ambientalmente viable; en la extracción de compuestos fenólicos evaluados, el proceso sin aprovechamiento energético es económicamente viable, mientras que el proceso con aprovechamiento energético es económica y ambientalmente viable, para un periodo mayor a 10 años. Se concluye que, el proceso de obtención de compuestos fenólicos con integración energética es recomendable como alternativa para el aprovechamiento de cáscara de plátano.

Palabras clave: cáscara de plátano; generación de electricidad; capacidad antioxidante; compuestos polifenólicos

\section{Evaluation of Alternatives for Energy and Bioactive Management of Plantain Peel}

\begin{abstract}
The objective of this work was to evaluate by simulation two alternatives for the use of green plantain peel for the generation of electric energy and for obtaining polyphenolic compounds. The technical, economic and environmental feasibility of the processes was determined; the simulation was fed with experimental data obtained in the characterization of banana peel with respect to its proximal, elementary, structural, thermogravimetric analysis, total phenolic content and antioxidant capacity. It was found that the shell can be used for the generation of energy at low cost and with a process that is environmentally viable; in the extraction of phenolic compounds the process without energy use is economically viable, while the process with energy use is economically and environmentally viable, for a period greater than 10 years. It is concluded that the process of obtaining phenolic compounds with energy integration is recommended as an alternative for the use of banana peel.
\end{abstract}

Keywords: plantain peel; electricity generation; antioxidant capacity; polyphenolic compounds 


\section{INTRODUCCIÓN}

El plátano es un tipo de planta herbácea monocotiledónea, que pertenece a la familia Musaceae (Davey et al., 2013). Son nativas del sudeste asiático, y sus variedades se clasifican según su composición genómica en Musa cavendish (Bananos) y Musa paradisíaca (plátanos) (Gibert et al., 2009). Hoy en día, el plátano se cultiva en al menos 107 países con una producción mundial de más de 76 millones de toneladas métricas (Olumba, 2014). Es considerado el cuarto cultivo más importante en el mundo, debido a que es un producto básico para la canasta familiar, su volumen de producción lo lleva a ser exportado, y como consecuencia de ello, ser una fuente de empleo e ingresos para la economía de algunos países del trópico y subtrópico. En Colombia se cultivan 11 variedades de plátano, ellas son: cachaco, dominico-hartón, dominico, guineo, hartón, maqueño, morado, pelipita, pompo, popocho, y trucho. El departamento de Caldas ubicado en la región centro andina de Colombia, motor de la transformación agroindustrial de la región genera una gran variedad de residuos, entre los que se encuentran las cáscaras de maracuyá, plátano, piña, tomate de árbol, mango, lulo y guanábana, las semillas de tomate de árbol, mandarina y naranja, el vástago de tomate de árbol, borra de café y cascará de plátano. Este último con un incremento importante debido al aumento en la producción de plátano (Cardona et al., 2012), que al año 2015 según el DANE alcanzó 1.310 .519 ton., lo cual generaron 445.576 ton. de cáscara. Parte de la producción de plátano en el departamento de Caldas es enviada a otros departamentos del país, otra parte es consumida directamente en los hogares y una parte es procesada en empresas de frituras. En Manizales, entre las empresas generadoras de cáscara de plátano están Jartru y Compañía S.A. y Comestibles Mapy S.A.S.

De la cosecha del racimo de plátano solo se utiliza del 20 al 30\% de su biomasa (Mazzeo et al., 2010), con un 70 a $80 \%$ por utilizar. La parte por utilizar está representada por el tronco de la planta con sus hojas, el brote floral, el raquis y la cáscara. La cáscara representa entre el $35 \%$ y el $40 \%$ del plátano, residuo potencialmente aprovechable en la fabricación de diferentes productos de valor agregado como es el almidón (Yusufu et al., 2014; Melo-Sabogal, 2015), abono verde y alimento para animales, harina para la elaboración de pastas alimenticias y productos de panificación (Mazzeo et al., 2010), pectinas útiles en la industria alimentaria, y productos elaborados en la industria del snack. A pesar de ello, la gran cantidad generada de estos residuos se han convertido en un problema para la industria en cuanto al almacenamiento y disposición final, para el ambiente porque son dispuestos en fuentes de agua y cerca de ellas, promoviendo la proliferación de plagas, malos olores, contaminación del suelo y del agua (Serna-Cock y Torres-León, 2014). Se ha reportado (Arun et al., 2015) que la harina de la cáscara de plátano tiene $5,89 \%$ de proteína, 5,12\% de grasa, $7,83 \%$ de ceniza y $11,03 \%$ de carbohidratos, y la cascara de plátano presenta vitaminas como ácido ascórbico $\left(0,08 \mathrm{mg} \cdot 100 \mathrm{~g}^{-1}\right)$, riboflavina $\left(0,065 \mathrm{mg} \cdot 100 \mathrm{~g} \mathrm{~g}^{-1}\right)$, niacina $\left(0,12 \mathrm{mg} .100 \mathrm{~g}^{-1}\right)$ y ácido fólico $\left(33,12 \mathrm{mg} \cdot 100 \mathrm{~g}^{-1}\right)$. También se han reportado (Agama-Acevedo et al., 2016) propiedades antioxidantes del pseudo tallo del plátano, aprovechables para la elaboración de productos con cualidades promotoras de salud, a pesar de reportarse una baja cantidad de polifenoles extraíbles en la cáscara de plátano verde, se reporta para ellos alta capacidad antioxidante. Esto hace que la cáscara de plátano sea considerada como un antioxidante natural.

Se ha considerado el uso de las cáscaras de plátano como materia prima para procesos de generación de energía debido a su relativo alto poder calorífico $(16,12 \mathrm{MJ} / \mathrm{kg})$ y contenido medio de cenizas $(9,92 \%$ base seca), en comparación con otras materias primas de origen biomásico (Fasina, 2014). También se ha encontrado que de las cascaras de plátano al ser pirolizadas se obtienen biosólidos o carbonizados con un poder calorífico superior alrededor de $22 \mathrm{MJ} / \mathrm{kg}$ (Ogunjobi y Lajide, 2015). Estos residuos también se han empleado para la producción de biogás mezclado con tuza de maíz y estiércol de vaca (Ukpabi et al., 2017), y para la producción de etanol empleando Saccharomyces cerevisia y Aspergillus spp (Stanley et al., 2017). El objetivo de este trabajo fue evaluar dos alternativas de aprovechamiento de la cáscara de plátano hartón verde para la generación de energía eléctrica y la obtención de compuestos polifenólicos por simulación empleando Aspen Plus. Cada una de estas alternativas se evalúa desde el punto de visto técnico, económico y ambiental. Para ello fue necesario alimentar la simulación con datos experimentales del análisis próximo, análisis elemental, análisis estructural, contenido de fenólicos totales, capacidad antioxidante y análisis termogravimétrico, de la cáscara de plátano hartón verde.

\section{METODOLOGÍA}

La metodología se presenta en tres subsecciones: en una se describe la obtención y adecuación de la materia prima; en la otra sección se describe los procedimientos utilizados en la caracterización fisicoquímica y en la última sección el procedimiento de simulación. 


\section{Obtención y pretratamiento del residuo}

Las cáscaras de plátano se obtuvieron de dos empresas de producción de frituras en la ciudad de Manizales, localizada a 2100 msnm, en el departamento de Caldas Colombia. Las cáscaras de plátano recolectadas eran de un plátano en la etapa de maduración 2, dado que el color de la cáscara era verde ( $\mathrm{pH}$ promedio de 6.14 \pm 0.3 y un porcentaje de acidez de $0.21 \pm 0.04 \%$ expresada para de ácido málico) y el color de la pulpa era blanco. El tiempo transcurrido entre la generación, obtención y almacenamiento de los residuos fue menor a 8 horas. Inmediatamente después del pelado del plátano, las cáscaras se sumergieron en una solución de eritorbato sódico $(1 \%)$ y ácido cítrico $(0,5 \%)$, con el fin de evitar el pardeamiento enzimático. Las cáscaras sumergidas en dicha solución se almacenaron a $-20^{\circ} \mathrm{C}$ con la finalidad de minimizar los cambios bioquímicos que se puedan presentar hasta el momento de realizar los análisis respectivos (Rúales, 2015). Con el fin de facilitar el proceso de presecado para los análisis, las cáscaras húmedas se redujeron de tamaño por medio de una cortadora de cuchillas. El presecado se realizó a $40^{\circ} \mathrm{C}$ hasta peso contante. Las cáscaras presecadas se redujeron de tamaño en un molino de discos hasta tamaños de partícula menores a $1 \mathrm{~mm}$.

\section{Caracterización fisicoquímica}

La caracterización fisicoquímica de las cáscaras de plátano se estableció a través del análisis próximo, análisis elemental, análisis estructural, contenido de fenólicos totales, capacidad antioxidante y análisis termogravimétrico.

\section{Análisis próximo y elemental}

El análisis próximo se determinó de acuerdo a las siguientes metodologías: la norma ASTM E871 para humedad, ASTM NREL/TP-510-42622 (Sluiter et al., 2005) para cenizas y ASTM E872 para materia volátil. El contenido de carbono fijo se estableció por diferencia. El análisis elemental experimental se llevó a cabo en un analizador específico para determinar carbono $(\mathrm{C})$, hidrógeno $(\mathrm{H})$ y nitrógeno $(\mathrm{N})$ según la norma ASTM D5373 - 08, y el azufre se determinó según norma ADTM D4239. Este análisis se realizó por triplicado.

\section{Análisis estructural}

La composición estructural de los residuos se determinó mediante la cuantificación de los compuestos extractivos (EXT), lignina (LGN), celulosa (CEL), hemicelulosa (HMC) y compuestos inorgánicos (cenizas). La preparación de los residuos para el análisis de la composición estructural se realizó siguiendo la norma NREL/TP-510-42620. Para determinar los porcentajes de CEL, HMC y LGN fue necesario someter los residuos previamente a dos extracciones Soxhlet utilizando agua y etanol como solvente, como indica la norma NREL/TP-510-42619. El porcentaje de lignina ácido insoluble (LGN) o lignina de Klason se determinó según la norma NREL/TP-510-42618. La determinación del porcentaje de holocelulosa (HLC) siguió la norma ASTM D1104, mientras que para cuantificar el porcentaje de celulosa se tomó la metodología descrita por Rowell et al., (2005). Para establecer el contenido de cenizas (CZ) se siguió la norma ASTM E1755-01. Estos análisis se realizaron por triplicado.

\section{Contenido de fenólicos totales y capacidad antioxidante}

La extracción de los compuestos fenólicos se llevó a cabo mediante maceración, agitación con vortex y centrifugación, la materia prima se seca y la maceración y molienda rompen la estructura celular para aumentar el área superficial del biomaterial en la extracción (Routray y Orsat, 2013). Los compuestos fenólicos totales se determinaron siguiendo la metodología descrita por Rúales (2015). Para este análisis se construyó una curva de calibración con la lectura de las absorbancias a $765 \mathrm{~nm}$ de 50,100,200, 350 y $500 \mathrm{mg} / \mathrm{l}$ del ácido gálico. Se utilizó agua destilada como blanco, la cual corresponde a la coordenada 0,0 en la curva de calibración. El resultado del análisis se remite a la curva de calibración y se expresa como miligramos equivalentes de ácido gálico (EAG) por g de muestra en base seca. Este procedimiento se realizó por triplicado y en ausencia de luz.

Para la determinación de la capacidad antioxidante se realizaron entre 5 y 7 diluciones del extracto metanólico, utilizando factores de dilución en un rango de 3 a 300 (Brand-Williams et al., 1995). La lectura de las absorbancias se realizó en un espectrofotómetro a $515 \mathrm{~nm}$ a diferentes tiempos (1, 15, 30 y 45 minutos), hasta que el valor de las absorbancias se estabilizó. Para la determinación del porcentaje de inhibición se tomaron las diluciones que presentaron inhibición entre 20 y 80 para construir un gráfico contra concentración con el fin de hallar la ecuación pertinente y calcular el IC50, considerado como la concentración de muestra necesaria para obtener una inhibición del $50 \%$ de los radicales libres (DPPH) (Rúales, 2015). 
Se sabe que los mecanismos de acción de los antioxidantes naturales incluyen la donación de átomos de hidrógeno, el atrapamiento o estabilización de radicales libres, la quelación de iones metálicos, entre otros (Achika et al., 2017). Esto indica que el estudio de la capacidad antioxidante de un producto natural debe llevarse a cabo explorando diversos métodos que abarquen varios de estos posibles mecanismos. Sin embargo, para analizar la alternativa de aprovechamiento bioactivo de las cáscaras de plátano hartón, el contenido de fenoles totales y capacidad antioxidante, se toman como una aproximación inicial del potencial bioactivo que tienen estas cáscaras. De tal manera que estos dos parámetros son suficientes para alimentar la simulación del proceso de extracción de compuestos fenólicos aquí estudiada (Rúales et al., 2017; Achika et al., 2017).

\section{Análisis termogravimétrico}

Después de secada la cáscara de plátano a $40^{\circ} \mathrm{C}$, se tomó una muestra entre 2 a $5 \mathrm{mg}$ para realizar el análisis de TGA a tres velocidades de calentamiento $\left(10,20\right.$ y $\left.30^{\circ} \mathrm{C} / \mathrm{min}\right)$ en atmósfera de nitrógeno, de acuerdo con la norma ASTM E2550-11. De este análisis se obtuvieron las curvas termogravimétricas (TG) y su derivada (DTG), de las cuales se determinaron las temperaturas características (temperatura inicial, pico y final) y los parámetros cinéticos de degradación en atmosfera inerte (proceso de pirólisis). A partir de los datos termogravimétricos se determinó la cinética de degradación térmica (triplete cinético: energía de activación $E$, factor de frecuencia - $A$ y orden de reacción $-n$ ) en atmosfera inerte por medio del método isoconversional de Starink (Gai et al., 2013 y Vyazovkin et al., 2001) y la teoría de Avrami Riutenberg (Ruitenberg et al., 2001) dadas por las Ecs. (1)-(2), respectivamente, así:

$$
\begin{aligned}
& \ln \left(\frac{\beta}{T^{1.8}}\right)=C_{S}-1.0037 \frac{E}{R T} \\
& \alpha=1-\exp \frac{-k(T)}{\beta^{n}} \\
& k(T)=A \exp \left(-\frac{E}{R T}\right) \\
& \alpha=\frac{W_{0}-W_{t}}{W_{0}-W_{\infty}}
\end{aligned}
$$

Donde $A$ es el factor de frecuencia o factor pre-exponencial, $C_{s}$ es una constante adimensional, $E$ es la energía de activación en $\mathrm{kJ} / \mathrm{mol}, k(T)$ es la constante de reacción dada por la ecuación de Arrhenius, $R$ es la constante universal de los gases que vale $8.314 \mathrm{~kJ} \mathrm{kgmol}^{-1} \mathrm{~K}^{-1}, T$ es la temperatura absoluta en $\mathrm{K}, W_{\infty}$ es la masa final de la muestra en $\mathrm{mg}, W_{0}$ es la masa inicial de la muestra en $\mathrm{mg}, W_{t}$ es la masa de la muestra en el tiempo $\mathrm{t}$ en $\mathrm{mg}$, $\alpha$ es la conversión y $\beta$ es la velocidad de calentamiento en $\mathrm{K} / \mathrm{min}$.

\section{Procedimiento de la simulación}

Para evaluar los potenciales aprovechamientos de la cáscara de plátano, se simularon tres procesos en las que se evaluaron las características económicas y ambientales. En el proceso 1 se simuló la generación de energía eléctrica (Fig. 1), mientras que en los procesos 2 y 3 se simuló la obtención de polifenoles (Figs. 23). En el proceso 2 se evaluó la obtención de polifenoles sin aprovechar la biomasa residual, que resulta de la de extracción de ellos, como combustible (Fig. 2). En el proceso 3 se evaluó la obtención de polifenoles aprovechando la biomasa residual como combustible (Fig. 3) a través de la integración energética dentro del mismo proceso.

El análisis técnico de los procesos se evaluó utilizando el software Aspen Plus, empleando el modelo termodinámico NTRL. La evaluación ambiental de los tres procesos se realizó empleando el algoritmo Reducción de Residuos (Waste Reduction - WAR GUI). Con esta herramienta se determinó el Potencial de Impacto Ambiental (PEI) teniendo en cuenta 4 categorías generales de impacto: Toxicidad humana, toxicidad ecológica, impacto global atmosférico e impacto regional atmosférico (Jaramillo, 2011). El programa WAR GUI importa directamente los resultados de la simulación del proceso realizados en Aspen Plus. Para este análisis, los sólidos lignocelulósicos se no consideraron ya que no representan un potencial de impacto ambiental al ser materiales biomásicos. 


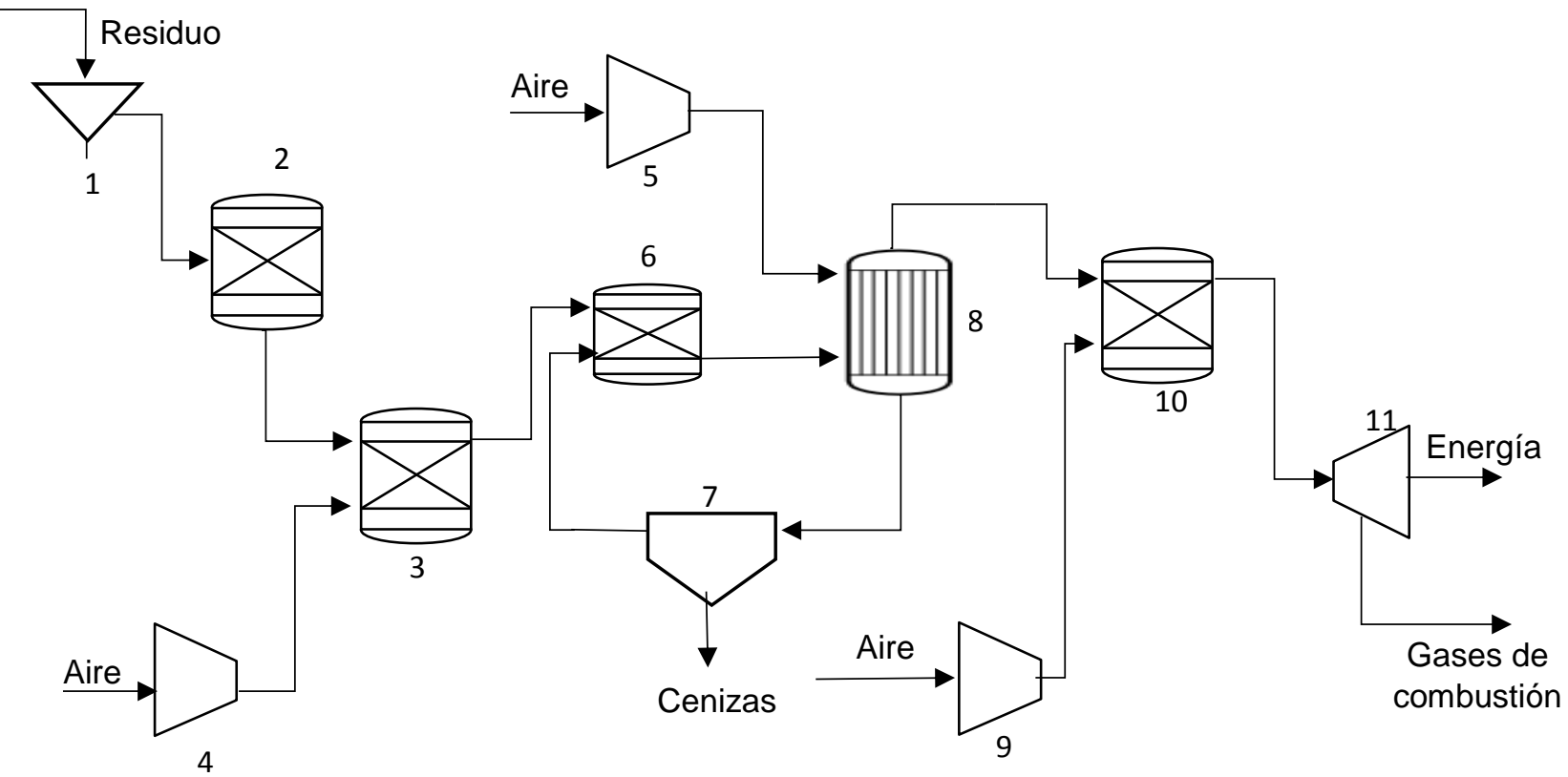

Fig. 1: Proceso 1: Diagrama de proceso de generación de energía eléctrica a partir de la cáscara de plátano. (1) Molino, (2) y (3) equipos de descomposición del residuo, (4) (5) (9) compresores, (6) pirolizador, (7) ciclón, (8) gasificador, (10) reactor de combustión de gases, (11) turbina

Las categorías de impacto evaluadas por el algoritmo WAR se dividen en dos grupos: atmosférica global y toxicológica global. Las categorías de impacto atmosférico global son: potencial de calentamiento global (GWP), potencial de agotamiento de ozono (ODP), potencial de acidificación o lluvia ácida (AP) y oxidación fotoquímica o potencial de formación de smog (PCOP). Las categorías de impacto toxicológico global son: potencial de toxicidad humana por ingestión (HTPI), potencial de toxicidad humana por inhalación o exposición dérmica (HTPE), potencial de toxicidad acuática (ATP) y potencial de toxicidad terrestre (TTP) (Quintero et al., 2006). Dos categorías se usaron para estimar el potencial de toxicidad humana: ingestión e inhalación/exposición dérmica. Estas dos categorías estiman el potencial de toxicidad porque consideran todas las rutas primarias de exposición de un producto químico.

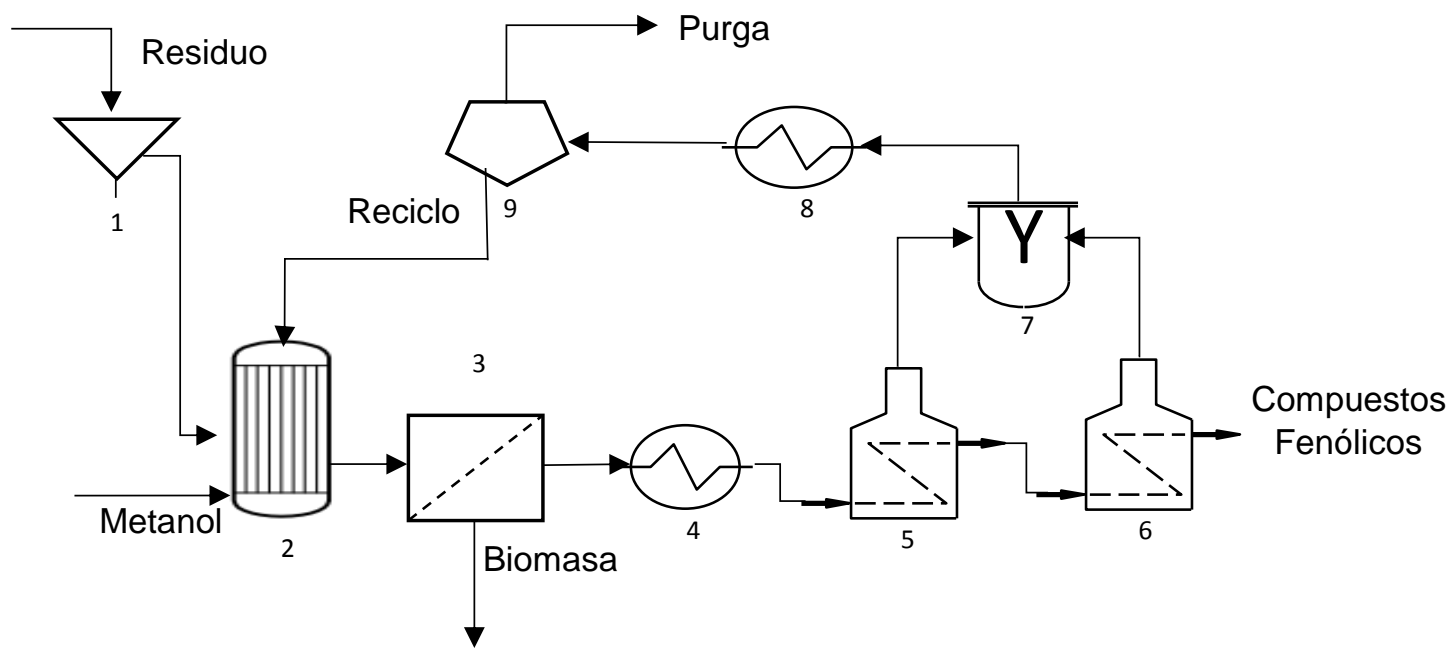

Fig. 2: Proceso 2: Diagrama de flujo para extracción de compuestos fenólicos con metanol como solvente y recuperación energética. (1) Molino, (2) extractor, (3) filtro, (4) y (8) intercambiadores de calor, (5) y (6) evaporadores, (7) mezclador, (9) divisor 


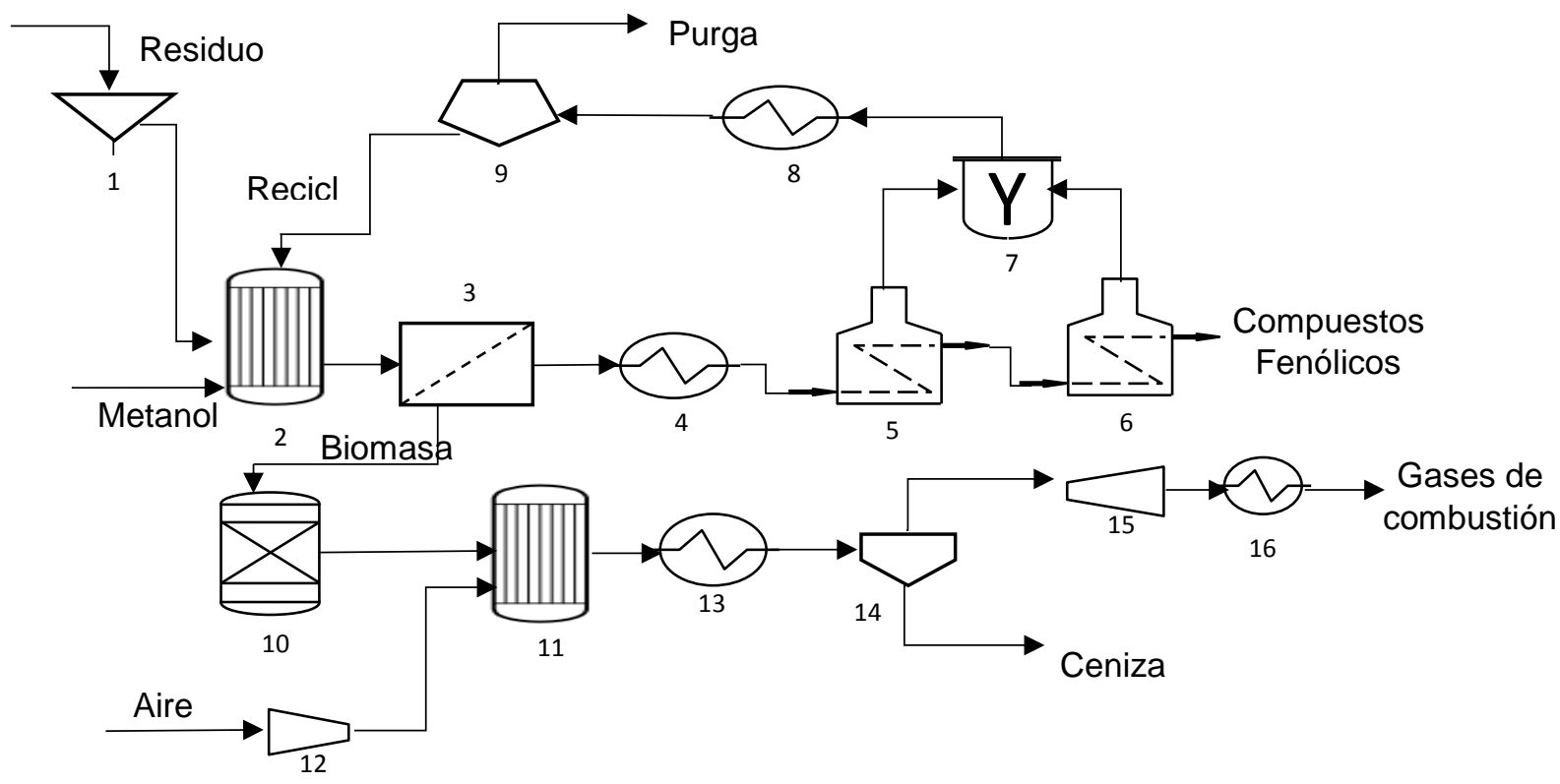

Fig. 3: Proceso 3: Diagrama de flujo para extracción de compuestos fenólicos con metanol como solvente y recuperación energética. (1) Molino, (2) extractor, (3) filtro, (4), (8), (13) y (16) intercambiadores de calor, (5) y (6) evaporadores, (7) mezclador, (9) divisor, (10) y (11) reactores de combustión, (12) compresor, (14) ciclón, (15) turbina

El análisis económico se llevó a cabo por medio de la herramienta incorporada en Aspen Plus llamada Aspen Process Economic Analyzer. Esta herramienta permitió determinar los costos totales en dólares que se requieren para montaje, operación y mantenimiento de un proceso para un periodo de 10 años, en el cual se trabajaran 8760 horas anuales. Estos resultados proporcionan información de la factibilidad económica de cada uno de los procesos planteados (Figs. 1-3). Para los costos de capital, esta herramienta tiene en cuenta los resultados de la simulación realizada en Aspen Plus. Los precios y las condiciones económicas tomadas para este análisis se muestran en la Tabla 1.

Tabla 1: Costos incluidos en el análisis económico (Datos tomados de Rúales, 2015)

\begin{tabular}{|l|r|}
\hline Característica & Costo \\
\hline Vapor de baja [USD/kJ] & $1,9 \mathrm{E}-06$ \\
\hline Refrigerante [USD/kJ] & $2,74 \mathrm{E}-06$ \\
\hline Operador [USD/h] & 2,56 \\
\hline Supervisor [USD/h] & 5,12 \\
\hline Electricidad [USD/kWh] & 0,115 \\
\hline Agua potable [USD/m $\left.{ }^{3}\right]$ & 0,4238 \\
\hline Combustible [USD/MWh] & 28,54 \\
\hline Metanol [USD/kg] & 3,38 \\
\hline Cáscara de plátano [USD/kg] & 0,15 \\
\hline Tasa de interés anual & $17 \%$ \\
\hline Tasa interna de retorno & $33 \%$ \\
\hline
\end{tabular}

En este análisis se buscó ajustar la cantidad de biomasa alimentada al proceso para alcanzar un margen de ganancia superior a un $30 \%$. Además, se tuvo en cuenta que el costo de los compuestos fenólicos en el mercado tiene un valor aproximado de 53,99 USD/kg (Rúales, 2015).

\section{RESULTADOS Y DISCUSIÓN}

En los resultados y discusión se presentan la caracterización fisicoquímica de la cascara de plátano; el aprovechamiento energético y bioactivo - Simulación y las alternativas potenciales del aprovechamiento de la cáscara de plátano 


\section{Análisis de composición de la cáscara de plátano}

En la Tabla 2 se presentan los resultados del análisis proximal, análisis elemental, análisis estructural y composición de fenoles totales y capacidad antioxidante de la cáscara de plátano, frente a algunos resultados de estos análisis reportados en la literatura. Al comparar los resultados de los análisis obtenidos, con lo reportado por otros autores, se observó, que no existe diferencia significativa entre los resultados para los análisis proximal y elemental, así mismo para los resultados de fenoles totales comparados con los valores reportados por Gimeno (2015) para residuos de industrias transformadoras de frutas tropicales. Sin embargo, el análisis estructural y bioactivo sí presenta diferencias significativas en los porcentajes de celulosa (20.90\%) y hemicelulosa (7.92\%) los cuales son menores que lo reportado por Tejada et al., (2014) para biomasa modificada con quitosano obtenida de residuos lignocelulósicos. La capacidad antioxidante experimental obtuvo valores menores a lo reportado por Gimeno (2015), debido a que la biomasa utilizada en los experimentos fue diferente y que las cascaras de frutas tienen mayor contenido de compuestos bioactivos que las del plátano. En la tabla $2{ }^{*}$ Valores calculados, ${ }^{* *}$ libre de extractivos.

Tabla 2: Caracterización fisicoquímica de la cáscara de plátano.

\begin{tabular}{|c|c|c|c|}
\hline & Experimental & Teórico & Referencia \\
\hline \multicolumn{4}{|c|}{ Análisis proximal- base seca } \\
\hline Humedad total - Hwt, \% & $84.94 \pm 0.10$ & 87.17 & \multirow{5}{*}{$\begin{array}{l}\text { (Colmenares, 2009) } \\
\text { Plátano verde clon } \\
\text { Hartón Común }\end{array}$} \\
\hline Materia volátil - MV, \% & $74.00 \pm 1.76$ & --- & \\
\hline Ceniza - Cz, \% & $8.18 \pm 0.43$ & 7.20 & \\
\hline Carbono Fijo - CF, \% & $17.82 \pm 1.18$ & --- & \\
\hline $\mathrm{MV} / \mathrm{CF}$ & $4.15 \pm 0.85$ & --- & \\
\hline Poder calorífico, MJ/kg & $17.39 \pm 1.27$ & 16.82 & $\begin{array}{c}\text { (Sheng et al., 2005) } \\
\text { Biomasa }\end{array}$ \\
\hline \multicolumn{4}{|c|}{ Análisis elemental, base seca libre de ceniza } \\
\hline Humedad al aire - Hw, \% & $15.06 \pm 0.05$ & --- & \multirow{8}{*}{$\begin{array}{l}\text { (Correa, 2016) } \\
\text { Biomasas residuales } \\
\text { provenientes de } \\
\text { cultivos tradicionales } \\
\text { en Colombia }\end{array}$} \\
\hline Carbono - C, \% & $48.40 \pm 0.21$ & 42.50 & \\
\hline Hidrógeno - H, \% & $7.27 \pm 0.01$ & 4.29 & \\
\hline Nitrógeno - N, \% & $1.36 \pm 0.01$ & 0.74 & \\
\hline Azufre - S, \% & $0.06 \pm 0.001$ & 0.02 & \\
\hline Oxígeno - O,\% & $42.91 \pm 0.18$ & 52.45 & \\
\hline $\mathrm{H} / \mathrm{C}$, base seca & $1.80 \pm 0.01$ & $1.21^{*}$ & \\
\hline $\mathrm{O} / \mathrm{C}$, base seca & $0.66 \pm 0.003$ & $0.67^{*}$ & \\
\hline \multicolumn{4}{|c|}{ Análisis estructural y bioactivo } \\
\hline Celulosa, \% & $42.87 \pm 0.23^{* *}$ & 20.90 & \multirow{3}{*}{$\begin{array}{c}\text { (Tejada et al., 2014) } \\
\text { Residuos } \\
\text { lignocelulósicos }\end{array}$} \\
\hline Hemicelulosa,\% & $29.09 \pm 0.43^{* *}$ & 7.92 & \\
\hline Lignina, \% & $10.91 \pm 0.01^{* *}$ & 18.11 & \\
\hline Extractivos totales, \% & $45.61 \pm 0.31$ & --- & \multirow{3}{*}{$\begin{array}{l}\text { (Gimeno, 2015) } \\
\text { Residuos industrias } \\
\text { transformadoras de } \\
\text { frutas tropicales }\end{array}$} \\
\hline Fenoles totales, mg AG/g & $15.4 \pm 0.80$ & 16.00 & \\
\hline $\begin{array}{l}\text { Capacidad antioxidante, } \\
\text { (mg Trolox/100g p.s.) }\end{array}$ & $161.10 \pm 50$ & $173.10 \pm 65$ & \\
\hline
\end{tabular}

\section{Análisis termogravimétrico de la cáscara de plátano}

En las Figs. 4 y 5 se presentan los resultados del análisis termogravimétrico de la cáscara de plátano a tres velocidades de calentamiento en atmosfera inerte. Las curvas TG de la Fig. 4 muestran que la temperatura inicial y final de descomposición presentan una leve diferencia en sus valores (ver Tabla 3). Lo cual se aprecia en el cambio de pendiente de las líneas horizontales al inicio y al final de cada curva TG, respectivamente.

Las curvas de la termogravimetría diferencial (DTG) se presentan en la Fig. 5. En esta figura se observa, entre 20 y $400^{\circ} \mathrm{C}$, dos picos. El primero de ellos representa la perdida de materia debido a la evaporación del agua contenida en la cáscara. El segundo pico indica que existe una sola etapa en un único mecanismo de reacción de degradación térmica. Esto está determinado por la composición química y física de la cáscara, lo cual juega un papel importante en su comportamiento en la pirólisis. Esta figura también muestra que la máxima velocidad de degradación de la cáscara de plátano disminuye al aumentar la velocidad de calentamiento. Además, la curva DTG se desplaza, alejándose del eje de las ordenadas con el aumento en la velocidad de calentamiento. 


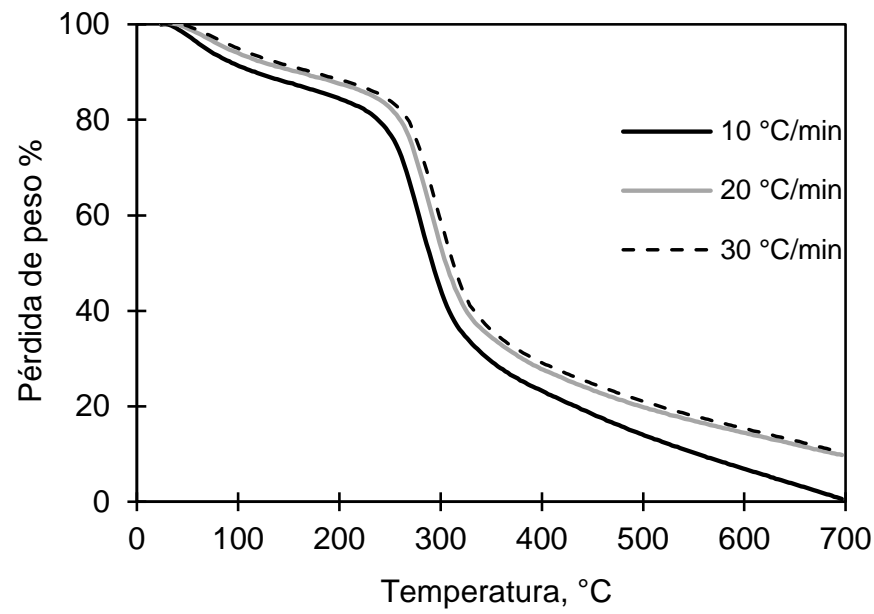

Fig. 4. Curvas termogravimétricas - TG de la cáscara de plátano a diferentes velocidades de calentamiento.

Esto implica que, al aumentar la velocidad de calentamiento, un mismo valor de conversión se alcanza a una mayor temperatura. Este comportamiento se ha explicado como un cambio en el mecanismo de reacción de descomposición con la velocidad de calentamiento (Park et al., 2000). El desplazamiento y la reducción de la altura de los picos en las curvas DTG, con el aumento en la velocidad de calentamiento, se atribuye a la reducción del tiempo de reacción debido a que se alcanza, en menor tiempo, la temperatura final de análisis. Similares resultados fueron reportados por (Slopiecka et al., 2012), para la pirólisis de madera de álamo. También se observa que la degradación térmica del $95 \%$ de la biomasa seca ocurre entre los 200 y $400^{\circ} \mathrm{C}$.

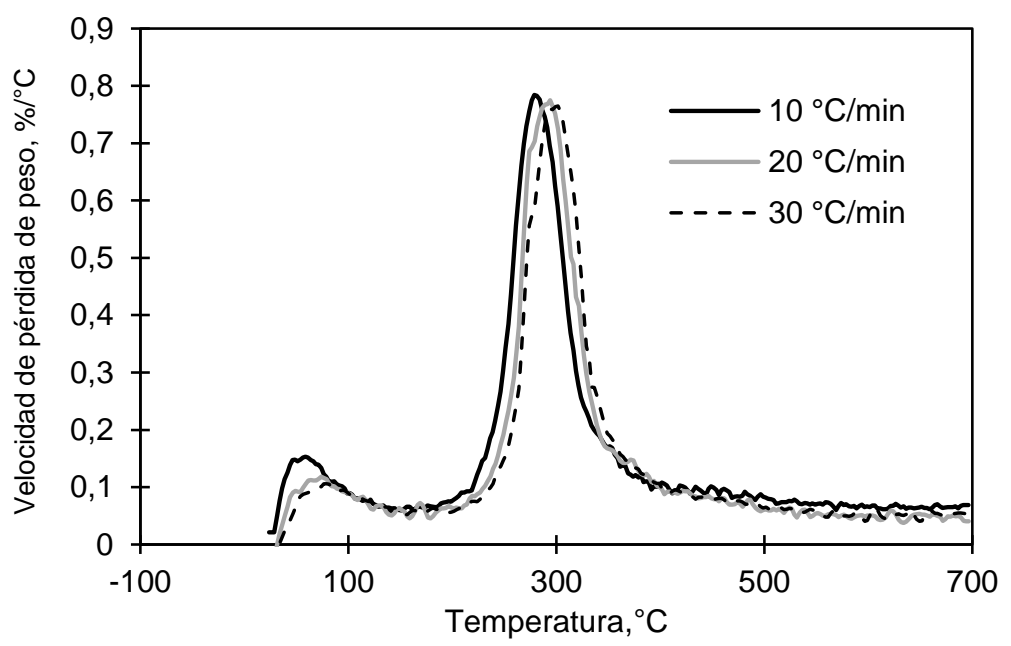

Fig. 5. Velocidad de pérdida de peso - DTG de la cáscara de plátano a diferentes velocidades de calentamiento.

En la Tabla 3 se presenta las temperaturas características y la máxima velocidad de pérdida de peso. En esta Tabla se corrobora el hecho que las temperaturas características (temperatura inicial, pico y final) de la cáscara de plátano aumentan con la velocidad de calentamiento, mientras que la máxima velocidad de pérdida de peso disminuye. Del análisis cinético empleando método isoconversional de Starink y la teoría de Avrami se encontró que la cáscara de plátano tiene una energía de activación de 219,21 $\pm 25,23 \mathrm{~kJ} / \mathrm{mol}$, un factor de frecuencia con Ln A de 40,55 $\pm 7,92$ y un orden de reacción de 0,20. El valor de energía de activación es similar al reportado por Giraldo y Montoya (2015) para la celulosa del aserrín de pino $(221,53 \mathrm{~kJ} / \mathrm{mol})$. Sin embargo, son valores diferentes al reportado Giraldo y Montoya (2015) para el tallo del banano con valores de energía de activación de $133.38 \mathrm{~kJ} / \mathrm{mol}$ y un valor del factor de frecuencia de Ln A de 14.78 .

Tabla 3: Parámetros característicos de las curvas termogravimétricas de la cáscara de plátano a diferentes velocidades de calentamiento.

\begin{tabular}{|l|c|c|c|}
\hline \multicolumn{1}{|c|}{ Parámetro característico } & $10^{\circ} \mathrm{C} / \mathrm{min}$ & $20^{\circ} \mathrm{C} / \mathrm{min}$ & $30^{\circ} \mathrm{C} / \mathrm{min}$ \\
\hline Temperatura inicial, ${ }^{\circ} \mathrm{C}$ & 184.12 & 205.21 & 218.34 \\
\hline Temperatura pico, ${ }^{\circ} \mathrm{C}$ & 279.56 & 291.25 & 299.89 \\
\hline Temperatura final, ${ }^{\circ} \mathrm{C}$ & 488.72 & 491.86 & 530.05 \\
\hline Máxima velocidad de pérdida de peso, $\% /{ }^{\circ} \mathrm{C}$ & 0.79 & 0.78 & 0.77 \\
\hline
\end{tabular}




\section{Aprovechamiento energético y bioactivo - Simulación}

Con el fin de determinar el análisis técnico y económico de los procesos, de midió la electricidad generada por la combustión de la cáscara de plátano la cual se obtuvo utilizando el flujo óptimo de entrada de aire en el gasificador una vez realizado el análisis de sensibilidad. Obtenido el punto óptimo dónde se cruza el consumo de metano con la producción de $\mathrm{CO}$ y $\mathrm{H}_{2}$, se tomó un punto medio de consumo de $\mathrm{CH}_{4}$. Con este valor se corrige la simulación a dicho punto el cual permite obtener la electricidad generada. Este punto óptimo tiene un flujo de aire en el gasificador de 6.39 ton/día, con una electricidad generada de $50.21 \mathrm{~kW}$.

Con el fin de determinar la eficiencia que puede ofrecer el proceso, la electricidad generada requiere tener en cuenta que en el proceso también se consumirá energía (ya sea térmica, mecánica o eléctrica). La electricidad total consumida de $27.34 \mathrm{~kW}$ representa la energía empleada por los compresores para ingresar el aire a los equipos. Con esta información se obtiene que la electricidad neta que se genera es de $22.84 \mathrm{~kW}$. Por lo tanto, la eficiencia porcentual de generación de energía eléctrica del proceso fue de $45.48 \%$ (kW electricidad neta por kW de electricidad generada). A partir del análisis económico se encontró el costo total para generar 50.21 kW de electricidad es de $\$ 290,985.86$ USD y con un precio de $\$ 0.0529$ USD $/ \mathrm{kW}$ con un margen de ganancia de $67 \%$.

En la Tabla 4 se muestra el balance de materia, para el proceso de extracción de compuestos fenólicos sin o con integración energética obtenidos a partir de simulación en Aspen Plus. Estos resultados son obtenidos buscando un margen de ganancia del $30 \%$ para que el proceso sea viable. Para el proceso de generación de energía se requiere $24100 \mathrm{~kg} / \mathrm{h}$ de aire y se producen $2131,91 \mathrm{~kg} / \mathrm{h}$ de cenizas y $30283,59 \mathrm{~kg} / \mathrm{h}$ de gases de combustión. La Tabla 5 presenta el análisis económico porcentual del proceso de extracción de compuestos fenólicos con aprovechamiento energético y el proceso de generación de electricidad. El costo de materia prima en la actualidad no tiene un valor económico en el mercado, dado que son residuos que se obtienen de diferentes empresas dedicadas a la producción de pulpa y confitería. Sin embargo, al costo de la materia prima (residuos) se asocian los costos de los fletes, para recoger y transportar los residuos de las distintas empresas generadoras hasta la planta de aprovechamiento de residuos. Estos costos son despreciables si la misma empresa que genera los residuos, los utiliza internamente para producir energía.

Tabla 4: Balances de materia para el proceso de extracción de compuestos fenólicos sin aprovechamiento energético

\begin{tabular}{|l|c|c|c|c|c|c|}
\hline \multirow{2}{*}{ Proceso } & \multicolumn{4}{|c|}{ Balance de materia } & \multirow{2}{*}{$\begin{array}{c}\text { Metanol total } \\
\text { utilizado } \\
{[}\end{array}$} \\
\cline { 2 - 8 } & \multicolumn{2}{|c|}{ Entra $[\mathrm{kg} / \mathrm{h}]$} & \multicolumn{3}{c|}{ Sale $[\mathrm{kg} / \mathrm{h}]$} \\
\cline { 2 - 7 } & Residuo & Metanol & Biomasa & Fenólicos & Purga & 68314.03 \\
\hline Sin integración energética & 10000 & 451.64 & 8315.50 & 24.88 & 2100.43 & 68.03 \\
\hline Con integración energética & 10000 & 441.45 & 0.00 & 24.88 & 2100.43 & 66700.00 \\
\hline
\end{tabular}

Tabla 5: Análisis económico porcentual de los procesos de extracción de compuestos fenólicos sin/con aprovechamiento energético y generación de electricidad

\begin{tabular}{|l|c|c|c|}
\hline \multicolumn{1}{|c|}{ Costos } & $\begin{array}{c}\text { Sin integración } \\
\text { energética }\end{array}$ & $\begin{array}{c}\text { Con integración } \\
\text { energética }\end{array}$ & $\begin{array}{c}\text { Generación de } \\
\text { electricidad }\end{array}$ \\
\hline Costos de materias primas & 72,86 & 49,58 & 5,84 \\
\hline Costos de utilidades & 17,04 & 38,41 & 17,56 \\
\hline Costos de operación & 8,97 & 9,85 & 43,87 \\
\hline Gastos de depreciación & 1,13 & 2,16 & 32,73 \\
\hline
\end{tabular}

\section{Análisis ambiental de los procesos}

Con la información obtenida en la simulación de los tres procesos por separado, se determina la contribución de los indicadores al potencial de impacto ambiental total, como se muestra en la Tabla 6 . El indicador que tiene la mayor influencia en el impacto ambiental de los procesos de extracción de compuestos fenólicos es el AP (Potencial de acidificación) con más del $85 \%$ del impacto. Mientras que la categoría ODP (Potencial de agotamiento de ozono) el PEI/h para cada uno de los procesos analizados tiene un valor de cero, esto se da porque en los gases que salen del proceso, no hay presencia de halógenos (como $\mathrm{F} \mathrm{y} \mathrm{Cl}$ ) que influyen directamente en el deterioro de la capa de ozono. Sin embargo, el proceso de aprovechamiento energético presenta dos principales indicadores que tienen la mayor influencia en el impacto ambiental potencial de formación de smog (POCP) y el Potencial de acidificación (AP). Para los índices HTPI, HTPE, TIP, ATP y GWP se tienen valores bajos de $\mathrm{PEI} / \mathrm{h}$. Estos valores bajos se presentan en los dos primeros procesos debido principalmente a la naturaleza del solvente utilizado, dado que el metanol es un compuesto tóxico, que puede generar daño en la salud humana y en los ecosistemas. Sin embargo, este impacto se minimiza realizando 
una recuperación y recirculación del metanol al proceso, considerando que se alcanzan recirculaciones entre el 90 y $98 \%$. Con esto se evita que el metanol sea liberado al ambiente generando daños a la vida acuática y el ambiente.

Los resultados obtenidos para el indicador GWP, se deben principalmente a la presencia de compuestos fenólicos y metanol en la corriente de purga; estas sustancias pueden generar compuestos orgánicos volátiles, que contribuyen en un $6,7 \%$ al calentamiento global. Para el proceso de generación de energía, los mayores valores de $\mathrm{PEI} / \mathrm{h}$ las categorías de impacto AP y POCP, se deben que en el proceso se producen compuestos azufrados como el $\mathrm{SO}_{2}$ y $\mathrm{SO}_{3}$, y óxidos de nitrógeno como el $\mathrm{NO}$ y $\mathrm{NO}_{2}$, que tienen un potencial de acidificación y formación de Smog alto por la deposición de ácidos resultantes a partir de su liberación. Esto influye directamente sobre la calidad de los suelos, atmósfera y aire, generando un impacto leve en la flora, fauna y salud humana. Los bajos valores de los índices HTPI, HTPE, TIP, ATP y GWP se encuentran relacionados con los gases que se generan en la gasificación y combustión, tales como $\mathrm{CO}, \mathrm{CH}_{4}$ y $\mathrm{CO}_{2}$, que influyen en estas categorías, principalmente en la categoría GWP, por ser considerados gases de efecto invernadero a excepción del CO.

Tabla 6: Contribución de los indicadores al potencial de impacto ambiental total del proceso de extracción de compuestos fenólicos

\begin{tabular}{|l|c|c|c|c|c|c|c|c|}
\hline \multirow{2}{*}{\multicolumn{1}{|c|}{ Proceso }} & \multicolumn{9}{c|}{ Indicador (\%) } \\
\cline { 2 - 11 } & HTPI & HTPE & TTP & ATP & GWP & ODP & PCOP & AP \\
\hline Sin integración & 2,07 & 0.03 & 2,07 & 3,82 & 2,78 & 0 & 3,24 & 86,02 \\
\hline Con integración & 1,19 & 0.02 & 1,19 & 4,02 & 2,93 & 0 & 0.01 & 90,67 \\
\hline Producción de energía & 0.42 & 0.61 & 0.42 & 0.03 & 0.82 & 0 & 52.31 & 45.39 \\
\hline
\end{tabular}

Comparando estos resultados con un proceso de producción de energía a partir de bagazo de caña (Michael et al., 2007) los valores para las categorías de potencial de toxicidad humana por ingestión (HTPI), potencial de toxicidad humana por inhalación o exposición dérmica (HTPE) y potencial de toxicidad terrestre (TTP), son similares. Esto está relacionado principalmente porque en ambos procesos de generación de energía no hay una gran emisión de compuestos tóxicos que afecten la salud humana. La principal diferencia entre lo reportado por (Sánchez et al., 2007) y el proceso de producción de energía está en el potencial de acidificación, ya que para el primer caso se tienen valores de AP cercanos a $0.1 \%$ y para el segundo caso, los valores de AP es de $45 \%$. Esta diferencia se debe especialmente a que en el proceso de producción de energía a partir de bagazo se presenta una etapa de cogeneración donde hay un mayor aprovechamiento de los gases de azufre para la producción de energía, mientras que para la producción de energía empleando gasificación y posterior combustión hay un mayor desprendimiento de gases de azufre y nitrógeno a la atmosfera.

\section{Alternativas potenciales del aprovechamiento de la cáscara de plátano}

Según los resultados obtenidos es posible determinar un aprovechamiento potencial para la cáscara de plátano. La cáscara de plátano tiene un porcentaje de humedad cercano al $15 \%$, esto indica que en un proceso de combustión se tiene un incremento de vapor de agua, el cual hace necesario un aumento en el consumo de energía para evaporar el agua en el interior de un proceso de combustión, esto afecta negativamente el poder calorífico. El contenido de cenizas cercano al $8 \%$ incrementa la probabilidad de que en un proceso termoquímico se tengan mayores depósitos en los equipos. El contenido de materia volátil cercano al $74 \%$, indica alta reactividad de la cáscara de plátano en la combustión, lo cual se corrobora con el análisis termogravimétrico donde la degradación se inicia alrededor de los $200^{\circ} \mathrm{C}$, pero termina cerca a los $400^{\circ} \mathrm{C}$. De igual manera estos se confirman con el bajo valor de la energía de activación obtenido por medio del método isoconversional de Starink. La baja temperatura final de degradación $\left(400^{\circ} \mathrm{C}\right)$ de la cáscara de plátano está relacionada con el bajo contenido de carbono fijo (9.34\%). Esto quiere decir, que la cáscara de plátano se puede emplear como combustible en un proceso de combustión, considerando que se debe someter a un proceso de secado, el cual puede ser al aire durante aproximadamente 5 días. Sin embargo, debido a su bajo poder calorífico, el cual depende del contenido de carbono fijo, se debe tener una alimentación continua de cáscara de plátano seca a la caldera. El contenido de nitrógeno y azufre en la cáscara de plátano, son bajos, esto indica que, al momento de su aprovechamiento como biocombustible, se tiene una baja producción de $\mathrm{NOx}$ y SOx, lo cual no afecta considerablemente el medio ambiente.

El contenido de celulosa en la cáscara de plátano plantea la posibilidad que puede ser utilizada como materia prima en una gran variedad de procesos, entre los que se destacan la industria del papel, textil, alimenticia y azúcares fermentables, y en la fabricación de biomateriales (Álvarez et al., 2012). Este residuo también podría derivarse en éteres y ésteres, los cuáles tienen aplicaciones como espesantes, estabilizantes, formadores de película, emulsificante, agente de suspensión, ligantes y como base para películas fotográficas, filtros de 
cigarrillos, marcos de gafas, juguetes y mangos de herramientas (Shokri et al., 2013) El contenido de hemicelulosa se puede aprovechar para la producción de furfural. El furfural es una plataforma de gran importancia en la industria química, pues a partir de este se elaboran productos como alcohol furfurílico, furfurilamina, ácido furoico y ácido furanacrílico, furano y tetrahidrofurano. De igual forma, la cáscara de plátano tiene potencial como materia prima en las industrias alimentaria, farmacéutica y química, ya que a partir de la hemicelulosa es posible producir ácido acético, compuesto ampliamente usado en dichas industrias. Tambien se podría emplear para la obtención de xilitol, el cual tiene un poder edulcorante similar a la sacarosa (Ramirez et al., 2012). Teniendo en cuenta que tanto la celulosa como hemicelulosa se pueden hidrolizar y ser sometidas a procesos fermentativos, la cáscara de plátano tiene un uso potencial en la industria de alcoholes. Una de las aplicaciones principales es la producción de bioetanol, que es uno de los biocombustibles de segunda generación más estudiados en los últimos años.

La cuantificación del contenido de compuestos fenólicos y la capacidad antioxidante de la cáscara de plátano, demostró que él extracto resultante es una buena fuente de estos, con valores de compuestos fenólicos $(15.4 \pm 0.80 \mathrm{mg} \mathrm{AG} / \mathrm{g})$ y actividad antioxidante $(161.10 \pm 50 \mathrm{mg}$ Trolox $/ 100 \mathrm{~g}$ p.s.); estos compuestos actualmente son muy utilizados en la industria como sustituto de componentes sintéticos y como ingredientes activos. Actualmente los residuos agroindustriales son una alternativa útil para la obtención de compuestos activos a partir de sustancias de origen natural y su procesamiento ha ido incrementando paulativamente, fomentando la obtención de productos con antioxidantes y de esta manera generando valor agregado a desechos agroindustriales. Los residuos de aceituna y de la industria vinícola han sido unos de los más utilizados para la extracción y estabilización de compuestos antioxidantes (Abd-ElGhany et al., 2010).

\section{CONCLUSIONES}

El programa permitió identificar que las categorías que aportan un mayor valor de $\mathrm{PEI} / \mathrm{h}$, son el potencial de formación de smog (PCOP) y potencial de acidificación o lluvia ácida (AP); esto se encuentra relacionado principalmente con los gases de azufre como: $\mathrm{SO}_{2}$ y $\mathrm{SO}_{3}$ y óxidos de nitrógeno que se generan en el proceso. Además, se encontró que la categoría de potencial de agotamiento de ozono (ODP) no genera ningún tipo de daño al medio ambiente, debido a la no presencia de compuestos halogenados en los gases efluentes del proceso. La determinación del precio de generación de kWh, permitió calcular un margen de ganancia del $67 \%$, indicando que es factible económicamente la implementación de este proyecto.

El proceso de extracción de compuestos fenólicos generó mayor margen de ganancia cuando no se considera el proceso de combustión para realizar el aprovechamiento energético. Sin embargo, la generación de residuos biomásicos hace necesario buscar otras técnicas de aprovechamiento. En cuanto al proceso de extracción de compuestos fenólicos sin integración energética, se encontró que los potenciales de impacto ambiental se deben principalmente a la naturaleza del solvente utilizado, el cual es tóxico; aun así, los valores del potencial de impacto ambiental para este proceso son significativamente bajos. Por otro lado, el proceso de extracción de compuestos fenólicos totales con integración energética y recirculación de solvente, presentó un menor potencial de impacto ambiental, debido a que los gases generados en este proceso se utilizan para la generación de energía, y el solvente se recupera y es recirculado al proceso.

\section{AGRADECIMIENTOS}

Los autores agradecen a la Universidad Nacional de Colombia sede Manizales por el apoyo económico al proyecto titulado "Identificación, cuantificación y caracterización de los residuos orgánicos generados en la agroindustria Caldense para su aprovechamiento energético y bioactivo", con código Hermes 35961.

\section{REFERENCIAS}

Abd-EIGhany, M.E., M.S. Ammar y A.E. Hegazy A.E., Use of Olive Waste Cake Extract as a Natural Antioxidant for Improving the Stability of Heated Sunflower Oil, ISSN: 1909 - 7891, World Appl. Sci. J., 11(1), 106-113 (2010)

Achika, J. I., R.G. Ayo, A.O. Oyewale y D.H. Jame, Chemical investigation and antioxidant activity of fractions of Lannea humilis (Oliv.) Engl, ISSN: 2149-0120, J. Turk. Chem. Soc., 4(2), 563-572 (2017)

Agama-Acevedo, E., J.A. Sañudo-Barajas y otros tres autores, Potential of Plantain Peels flour (Musa paradisiaca L.) as a Source of Dietary Fiber and Antioxidant Compound, doi.org/10.1080/19476337.2015.1055306, CyTA - J. Food., 14(1), 117-123 (2016)

Álvarez, A., R. Salgado, E. García y otros 8 autores, Aprovechamiento Integral de los Materiales Lignocelulósicos, ISSNe: 0121-6651, Rev. Iberoam. Polím., 13(4), 140-150 (2012)

Arun, K.B., F. Persia, P.S. Aswathy y otros siete autores, A Potential Source of Antioxidant Dietary Fibre for Developing Functional Cookies, doi.org/10.1007/s13197-015-1727-1, J. Food Sci. Technol., 52(10), 6355-6364 (2015) 
Brand-Williams, W., M.E. Cuvelier y C. Berset, Use of Free Radical Method to Evaluate Antioxidant Activity, doi.org/10.1016/S0023-6438(95)80008-5, Lebensmittel-Wissenschaft \& Technologie, 28, 25 - 30 (1995)

Cardona, C.A., C.E. Orrego y J.A. Tamayo, Una Oportunidad para el Desarrollo de la Agroindustria en el Departamento de Caldas, Primera Ed. Manizales, Univ. Nacional de Colombia, Colombia (2012)

Colmenares, M.J., Elaboración de Harina de Pulpa y Cáscara ye Plátano Verde Clón Hartón Común para la Formulación de una Mezcla de Harina para Arepas a Base de Plátano y Maíz, Trab. de grado, Univ. Central de Venezuela, Venezuela (2009)

Correa, S.A., Análisis Teórico de la Combustión de Biomasa Residual Proveniente de Cultivos Tradicionales en Colombia, Trab. de grado, Univ. Tecnológica de Pereira, Facult. de Ing. Mec., Colombia (2016)

Davey, M.W., R. Gudimella y otros tres autores, A Draft Musa Balbisiana Genome Sequence for Molecular Genetics in Polyploid, Inter- and Intra-Specific Musa Hybrids, doi.org/10.1186/1471-2164-14-683, BMC Genomics 14(683), 6 -20 (2013)

Fasina, O., Energy Potential of Yam and Plantain Peels, ISSN: 1682-1130, Agric Eng Int: CIGR Journal, 16 (2), $53-58$ (2014)

Gai, C., Y. Dong y T. Zhang, The Kinetic Analysis of the Pyrolysis of Agricultural Residue under Non-Isothermal Conditions, doi: 10.1016/j.biortech.2012.09.089, Bioresource Technol., 127, 298-305 (2013)

Gibert, O. y otros 8 autores, Differentiation between Cooking Bananas and Dessert Bananas. 1. Morphological and Compositional Characterization of Cultivated Colombian Musaceae (Mus sp.) In Relation to Consumer Preferences, doi: 10.1021/jf901788x, J. Agric. Food Chem. 57(17), 7857-7869 (2009)

Gimeno, D., Optimización de la Extracción de Compuestos de Interés en Subproductos de Frutas Tropicales para su Aplicación en Matrices Alimentarias, Ed. Zaragoza, Universidad de Zaragoza, España (2015)

Giraldo, N.I. y N. Montoya, Caracterización de Residuos de Banano (Pseudotallo y Hojas) Mediante Análisis Termogravimétrico para Uso Potencial como Biocombustible Sólido, Trab. de grado, Facult. de Ing., Univ. de Medellín (2015)

Jaramillo, J.J., Evaluación Tecno-Económica de la Producción de Biocombustibles a Partir de Microalgas, M.Sc. thesis, Dept. Ing. Qca., Univ. Nacional de Colombia Sede Manizales, Colombia (2011)

Mazzeo, M., L.L. Agatón y otros tres autores, Aprovechamiento Industrial de Residuos de Cosecha y Poscosecha del Plátano en el Departamento de Caldas, doi.org/10.26507/rei.v5n9.14, Rev. Educ. en Ing, Asociación Colombiana de Facultades de Ingeniería-ACOFI 9, 128-139 (2010)

Melo-Sabogal, D.V., Y. Torres-Grisales, J. A. Serna-Jiménez y L.S. Torres-Valenzuela, Aprovechamiento de Pulpa y Cáscara de Plátano (Musa paradisiaca Spp) para la Obtención de Maltodextrina, doi:10.18684/BSAA(13)76-85, Biotecnol. Sect. Agrop. y Agroind. 13(2), 76-85 (2015)

Michael W., W. May y H. Hong, Life-Cycle Energy and Greenhouse Gas Emission Impacts of Different Corn Ethanol Plant Types, doi:10.1088/1748-9326/2/2/024001, Environ. Res. Lett. 2, 1-13 (2007)

Ogunjobi J.K. y L. Lajide, The Potential of Cocoa Pods and Plantain Peels as Renewable Sources in Nigeria, doi.org/10.1080/15435075.2013.848403, Inter. J. Green Energy., 12(4), 440-445 (2015)

Olumba, C.C., Productivity of Improved Plantain Technologies in Anambra State, Nigeria. doi:10.5897/ajar2014.8891, Afr. J. Agric. Res., 9(29), 2196-2204 (2014)

Park, J.W., S.C. Oh y otros tres autores, Kinetic Analysis of Thermal Decomposition of Polymer Using a Dynamic Model, ISSN-e 1975-7220, Korean J. Chem. Eng. 17(5), 489-496 (2000)

Quintero, J.A., M.I. Montoya, O.J. Sánchez y C.A. Cardona, Evaluación del Impacto Ambiental del Proceso de Obtención de Alcohol Carburante Utilizando el Algoritmo de Reducción de Residuos, ISSN: 0120-6230, Rev. Fac. de Ing. 36, 85-95 (2006)

Ramírez, K., Ó. Rojas y otros tres autores, Obtención de Xilosa a Partir de Desechos Lignocelulósicos de da Producción y Proceso Industrial de la Piña (Ananas comusus), ISSN: 1011-0275, Uniciencia, 26, 75-89 (2012)

Routray, W. y V. Orsat, Preparative Extraction and Separation of Phenolic Compounds. In: Ramawat K., Mérillon JM. (eds), 1a Ed., 2013-2045, Springer, Berlin, Alemania, (2013)

Rowell, R.M., R. Pettersen y otros tres autores, Cell wall chemistry. In: Rowell, R. M. (Ed.). Handbook of wood chemistry and wood composites. CRC Press, Boca Raton, Florida. USA. 35-74. (2005)

Ruales, A.V., A. F. Rojas y C.A. Carona, Obtención de Compuestos Fenólicos a Partir de Residuos de Uva Isabella (Vitis labrusca). doi.org/10.18684/bsaa(v15) EdiciónEspecialn2.580, Biotecnología en el Sector Agropecuario y Agroindustrial, 15(2), 79-72 (2017)

Ruales, A.V., Evaluación del Potencial Energético y Bioactivo de los Residuos Generados por la Producción y Transformación de la Uva, M.Sc. thesis, Dept. Ing. Qca., Univ. Nacional de Colombia sede Manizales, Colombia (2015)

Ruitenberg, G., E. Woldt y A.K. Petford-Long, Comparison the Johnson-Mehl-Avrami-Kolmogorov Equations for Isothermal and Linear Heating Conditions, doi.org/10.1016/S0040-6031 (01)00584-6, Thermochim. Acta, 378, 97-105 (2001) 
Sánchez, O.J., C.A. Cardona y D.L. Sánchez, Análisis de Ciclo de Vida y su Aplicación a la Producción de Bioetanol: Una Aproximación Cualitativa, ISSN: 2344-8172, Rev. Univ. EAFIT. 43, 59-79 (2007)

Serna-Cock, L. y C. Torres-León, Potencial Agroindustrial de Cáscaras de Mango (Mangifera indica L.) Variedades Keitt y Tommy Atkins, doi.org/10.15446/acag.v64n2.43579, Acta Agron. 64(2), 110-115 (2014)

Sheng, C. y J.L. Azevedo, Estimating the Higher Heating Value of Biomass Fuels from Basic Analysis Data, org/10.1016/j.biombioe.2004.11.008, Biomass Bioenerg., 28, 499-507 (2005)

Shokri, J. y K. Adibkia, Application of Cellulose and Cellulose Derivatives in Pharmaceutical Industries, in Cellulose Medical, Pharmaceutical and Electronic Applications. Ed. Irán: Theo Van de Ven and Louis (2013)

Slopiecka, K., P. Bartocciy y F. Fantozzi, Thermogravimetric Analysis and Kinetic Study of Poplar Wood Pyrolysis, doi: 10.1016/j.apenergy.2011.12.056, Appl. Energ. 97, 491-497 (2012)

Sluiter, A., B. Hames y otros cuatro autores, Determination of Ash in Biomass (NREL/TP-510-42622), National Renewable Energy Laboratory (2005)

Stanley H.O., R. Ofuru y C.D. Onwukwe, Production of Bioethanol from Plantain Peels Using Aspergillus spp. And Saccharomyces spp, ISSN: 1118-1931, Scientia Africana, 16(1), 5-13 (2017)

Tejada, L., C. Tejada, W. Marimón y Á. Villabona, Estudio de Modificación Química y Física de Biomasa (Citrus sinensis y Musa paradisiaca) para la Adsorción de Metales Pesados en Solución, ISSN: 1909-2474, Luna Azul, 39,124-142 (2014)

Ukpabi, C., O. Ndukwe y otros tres autores, The Production of Biogas using Cow Dung and Food Waste, doi:10.5923/j.jmc.20170702.01, Inter. J. Mat. Chem. 7(2), 21-24 (2017)

Vyazovkin, S., A.K. Burnham y otros cuatro autores, ICTAC Kinetics Committee Recommendations for Performing Kinetic Computations on Thermal Analysis Data, doi.org/10.1016/j.tca.2011.03.034, Thermochim. Acta, 520, 1-19 (2001)

Yusufu, P.A., T. B. Mosiko y O.O. Ojuko, Effect of Firm Ripe Plantain Fruit Flour Addition on the Chemical, Sensory and Microbial Quality of Fura Powder, doi.org/10.1016/S0189-7241(15)30094-1, Nigerian Food J. 32(1), 38-44 (2014) 
